\section{Pharmaco-economic study and cost of care for chronic diseases: Case of Haemophilia in Morocco}

\author{
Khairoun C*, Khorassani M, Kili A, Hssissen L, Kababri M and \\ Khattab M \\ Center of Pediatric Hematology and Oncology in rabat, Children's Hospital, Faculty of Medicine \\ and Pharmacy, Mohammed V University of RABAT, Morocco
}

\section{Abstract}

This paper is an attempt to enrich the literature about the role that can play some economic approaches, such as cost-effectiveness analysis, in order to help medical stuffs to decide about the treatment to adopt in case of a chronic disease such as Haemophilia. Data from morocco were gathered in order to explain the importance of such approaches.

\section{Introduction}

The economist can claim to make an original contribution to the evaluation of actions undertaken in the health field by the introduction of the issue of economic rationality in the decision-making process. Economic theory is, indeed, a theory of choices putting the results of alternative actions into relation with the means they require respectively. The objective sought is efficiency, that is to say, the best ratio between the cost of the resources mobilized and the results obtained. Applied to the health field, the economic approach is distinguished from both a medical approach to evaluation, such as "evidence-based medicine", which favours the single criterion of effectiveness, and from an accounting or financial approach which emphasizes the expenditures generated by health actions. The implementation of this problem of efficiency requires the development and adaptation of economic calculation tools and generally takes the form of a cost-effectiveness analysis (CEA), cost-utility analysis (ACU) or cost-benefit analysis (CBA) which constitute the fundamental tools of the toolbox for the economic evaluation of health strategies. This economic approach to health decision-making is now widely used to inform the choices to be made in terms of resource allocation.

The purpose of this paper is to highlight the importance of this type of study in the treatment of chronic diseases, especially in case of Haemophilia. These analyses are necessary to assess the real cost of drugs and the benefits of their use in comparison with prophylactic treatment strategies.

\section{More Information}

*Address for Correspondence: Dr. Chaimae Khairoun, Center of Pediatric Hematology and Oncology in rabat, Children's Hospital, Faculty of Medicine and Pharmacy, Mohammed V University of RABAT, Morocco,

Tel: +212624800879;

Email: chaimaekhairoun1@gmail.com

Submitted: 13 March 2020

Approved: 01 April 2020

Published: 02 April 2020

How to cite this article: Khairoun $C$, Khorassani M, Kili A, Hssissen L, Kababri M, Khattab M. Pharmaco-economic study and cost of care for chronic diseases: Case of Haemophilia in Morocco. J Adv Pediatr Child Health. 2020; 3: 024-026.

DOI: 10.29328/journal.japch.1001013

Copyright: (c) 2020 Khairoun C, et al. This is an open access article distributed under the Creative Commons Attribution License, which permits unrestricted use, distribution, and reproduction in any medium, provided the original work is properly cited.

Keywords: Pharmaco-economic study; Costeffectiveness analysis; Haemophilia; Prophylaxis

Check for updates

OPEN ACCESS

\section{Literature review}

Chronic diseases are defined as long-term, progressive pathologies, often associated with disability and the threat of serious complications. A chronic disease is therefore characterized by a slow progression, with no tendency to cure and with a decrease in quality and life expectancy [1]. According to the World Health Organization (WHO), its main risk factors are four in number: tobacco, alcohol, physical inactivity and unhealthy eating habits [2]. Its prevention would, in terms of public health, considerably reduce morbidity and mortality.

For the person suffering from chronic disease, the illness "is above all, a chronic relation to medicine, a "chronification" of medical follow-up which requires on the part of the sick person, a continuous adaptation to this specific organization of care. This presupposes that professional, family and social life can be reconciled with medical follow-up, at the cost, most of the time, of real adjustment work" [3].

According to a study done in Belgium, patient with a chronic disease represent $25 \%$ of the population and $70 \%$ of total health expenditure. The considerable and growing weight of chronic conditions on public health expenditure has 
increased over the years. In just 5 years, these expenses have increased by $10 \%$ [4].

Haemophilia is a good example of a chronical illness. It is an inherited haemorrhagic disease with recessive inheritance linked to the $\mathrm{X}$ chromosome. The biological diagnosis is made on an extension of the TCA with a factor VIII deficiency for haemophilia A and factor IX for haemophilia B.

There is a good correlation between the intensity of the anti hemophilic factor deficiency and the clinical severity of the disease [5-11]. We thus define:

- Severe haemophilia: Factors VIII or IX: $<1 \%$,

- Moderate haemophilia: Factors VIII or IX: 1 to 5\%,

- Minor haemophilia: Factors VIII or IX: 6 to $35 \%$

Hemophilia affected 1 case in 10,000 births of which $80 \%$ are haemophiliacs A (haemophilia due to factor VIII deficiency) and 20\% haemophiliacs B (haemophilia due to factor IX deficiency) [12]. The World Health Organization (WHO) estimates that there are currently 400,000 cases of haemophiliacs worldwide.

We do not have the current state of statistical data on haemophilia in Morocco. If we consider the statistics, which estimate that one child in 10,000 suffers from haemophilia, we must have in Morocco 3700 children suffering from this anomaly while the country identifies only 950 cases at the level AMH (Moroccan association of haemophiliacs). This major shortcoming, due to the absence of a national haemophilia registry, explains the insufficient level of therapeutic care for patients.

\section{Methodology}

We used the cost-effectiveness analysis during our study. A cost-effectiveness analysis in the strict sense compares the costs and effects of one health intervention with another. It is a measure of the marginal impact of a choice on costs and effects. The analysis is summarized in cost-effectiveness, where the numerator is an estimate of the difference in costs between two strategies, and the denominator is an estimate of the difference in expected results by choosing one intervention over another (Figure 1). The results of the intervention can be expressed in the number of hospitalizations avoided, the number of cases cured, the number of lives saved or the number of years of life saved. The results are often expressed in years adjusted for quality (Quality Adjusted Life Years, QALY). This is called cost-utility analysis. One way to take quality of life into account is to ask the study participant to give a score of 0 to 1 for their current state of health. A person in perfect health will give the score of 1 and a person with a reduced quality a lower score, for example of 0.8 . The zero score is reserved for the worst-case scenario as well as for deceased patients. Thus, a year of life of the person in poor health will be worth, according to this example, 0.8 QALY and two years in this state, 1.6 QALY. Another way to assess patients' quality of life is to submit questionnaires examining different aspects of their lives.

\section{Results}

To highlight the importance of prophylaxis in the case of haemophilia, we collected data from 12 patients followed up in the Center of Pediatric Hematology and Oncology in rabat, Morocco, in order to trace exactly the costs of this treatment table 1.

The current challenge of treatments is to preserve the joints of haemophiliac patients, particularly affected during repeated bleeding, as soon as possible. In order to preserve joint capital, a "zero bleeding" objective is targeted. However, this objective can only be imagined if each treatment is personalized, because each haemophiliac patient is unique. In other words, the prophylactic treatment must be adapted to each patient, considering his age, his hemorrhagic phenotype, his lifestyle (personal, professional), his level of physical activity, but also its pharmacokinetic parameters [13].

\section{A: New activity being evaluated B: Standard of care}

Incremental cost-effectiveness ratio (ICER):

$$
I C E R=\frac{\text { Cost } \mathrm{A}-\text { Cost } \mathrm{B}}{\text { Effects } \mathrm{A}-\text { EFFects } \mathrm{B}}
$$

Figure 1: Incremental cost-effectiveness ratio.

\begin{tabular}{|c|c|c|c|c|c|c|c|}
\hline Name & Birth date & City & Type A/B & PAH & DDT & Dose range UI/Kg/total & Indication \\
\hline CY & $19 / 05 / 97$ & Errachidia & $A$ & Factane & $22 / 03 / 2011$ & 1500 & Knee and elbow arthropathy \\
\hline $\mathrm{OM}$ & $10 / 02 / 96$ & El hajeb & A & Factane & $24 / 01 / 2011$ & 500 & Knee arthropathy \\
\hline B Y & 20/09/07 & Kénitra & A & Factane & $10 / 02 / 2010$ & 1000 & Knee hemarthrosis \\
\hline ST & $09 / 02 / 98$ & Témara & A & Kogenate & $30 / 05 / 2011$ & 500 & Hemorrhagic episode of the shoulder \\
\hline LA & 20/01/03 & Salé & A & Kogenate & $12 / 09 / 2012$ & 1000 & Gou hemarthrosis \\
\hline M R & 26/07/95 & Skhirate & B & Betafact & $15 / 08 / 2012$ & 2000UI & Knee hemarthrosis \\
\hline FA & $11 / 12 / 0 \mathrm{e} 7$ & Skhirate & B & Betafact & $23 / 08 / 2012$ & $1000 U 1$ & Knee hemarthrosis \\
\hline A A & $31 / 08 / 03$ & Skhirate & B & Betafact & $25 / 09 / 2012$ & $1000 \mathrm{UI}$ & Knee and elbow hemarthrosis \\
\hline B M & & Tiflet & A & Kogenate & $02 / 10 / 2012$ & $2000 \mathrm{UI}$ & Knee hemarthrosis \\
\hline M A & 01/10/08 & Khmissate & A & Kogenate & $28 / 12 / 2011$ & $500 \mathrm{UI}$ & Knee hemarthrosis \\
\hline $\mathrm{HM}$ & 2002 & Agadir & A & Kogenate & $10 / 09 / 2012$ & $1000 \mathrm{UI}$ & Hemarthrosis of both knees \\
\hline $\mathrm{HH}$ & $10 / 03 / 91$ & Tamara & A & Kogenate & $04 / 10 / 2012$ & $2000 U 1$ & Hemarthrosis of the knee and wrist \\
\hline
\end{tabular}


For the case of our patients:

The weight of the patients varied from 19 to $80 \mathrm{~kg}$.

The average dose was $30 \mathrm{IU} / \mathrm{kg}$,

With a variable of 15 to $50 \mathrm{IU} / \mathrm{kg}$ per week.

- The total annual consumption of clotting factor concentrates is 936,000 IU of factor VIII and 576,000 IU of factor IX.

- The average factor consumption is $1757 \mathrm{IU} / \mathrm{kg} /$ patient/ year (1300 IU -2700 IU)

With this "personalized" protocol, we were able to

- Reduction, see disappearance of hemarthroses

Resumption of normal activity

Reduction of disability

And above all the Reduction of cost

\section{Discussion}

The cost of treatment is the main obstacle to long-term prophylaxis [14]. Prophylaxis is only possible if significant resources are allocated to haemophilia care. However, it is profitable in the long term because it eliminates the very high cost inherent in the subsequent management of joint damage and improves the quality of life [15]. It is very important to collect and provide scientific evidence justifying the high cost of treatment, even in countries that have traditionally invested in haemophilia care and where prophylaxis is well established as a therapeutic standard. In countries where access to clotting factor concentrates is limited, prophylaxis is out of reach for the majority of patients. However, some countries are now starting to give prophylactic treatment to children [16]. The follow-up data of these patients will be very important in order to confirm the effects on their joints. Patients and healthcare professionals must work together to advocate for a national haemophilia care program and the purchase of sufficient clotting factor concentrates.

In Morocco, the theoretical annual cost of a prophylactic treatment for a haemophiliac patient varies according to the weight, the severity of the disease and the appearance of complications. This cost varies from 700,000 to $3,000,000$ $\mathrm{MDH}$.

The true annual cost of factor concentrates varies between 350,000 MDH and 1,500,000 MDh, which represents a drop of $50 \%$ compared to theoretical figures. To be able to conduct a true cost-effectiveness analysis, figures on the costs of treatments on demand for haemophilia (direct, indirect, medical and non-medical) must be available to us to prove the effectiveness of prophylaxis in case of haemophilia. Prophylaxis reduces the incidence and severity of joint bleeding, preserves joint function, and improves quality of life. It is therefore profitable in the long term since it eliminates the high cost of the subsequent care of patients whose joints are damaged. Cost-benefit studies to establish the minimum dosage are needed to lower the cost of care and expand access to prophylaxis on a larger scale.

\section{Conclusion}

Analysing the cost of drugs and care is becoming essential for the health system, particularly in countries with limited resources. Care protocols for recommendations must be implemented, disseminated, evaluated and monitored. The prescription and dispensation of antibiotics, particularly in paediatrics, deserve a pharmaco-economic study. A comprehensive drug analysis is necessary to assess the actual cost of the drugs and the benefits of their use. Executively expensive drugs are cheaper in real cost paradoxically, others accessible to the general population, not very expensive, are more expensive in real cost.

\section{References}

1. Ministry of Health. National Chronic Disease Plan 2007-2011 [Internet] 2007.

2. World Health Organization. WHO: Chronic diseases.

3. Carrier S, Morin P, Gross $\mathrm{O}$, De La Tribonnière X. L'engagement de la personne dans les soins de santé et services sociaux: Regards croisés France-Québec: Presses de l'Université du Québec. 2017.

4. https://www.mloz.be/sites/default/files/publications/envol_cout_ maladies_chroniques_0318.pdf

5. Hemophilia B in Morocco. Current state and outlook.

6. Emmanuil Kombarelis, willim P. J Clini Epidemiology. 2011; 47.

7. Hay CR, Brown S, Collins PW. le diagnosticet la gestion du facteur VII et IX,Centre d'hémophilie Br, Haematol. 2006; 133: 591-605.

8. Jenny Goudemand, Yves Laurien. 'hémophilie Orphanet Mai. 2006.

9. Kessler CM, Goldman L. Hemmoragic disorders :coagulation factor diferences,cecil Medecine 23rd ed,Philadelphia, Sauders Elsevier. $2007 ; 180$.

10. Kessler CM, Mariani. manifestations cliniques et traitement de l'hémophilie,Hemostasis et Thrombosis 5èd Philadelphia. 2006; 887.

11. Khachidz M, Buil A, Viel KR, Porter S. Genetic determinants of normal variation in coagulation factor IX. Hemostasis Thrombosis. 2006; 4 1537-1545.

12. Siegmund B, Richter $H$, Pollmann $H$. Need for prophylactic treatment in adult haemophilia A patients. Transfusion Medicine and Hemotherapy. 2009; 36: 283-288.

13. Suzuki Katsuhiko. Chronic inflammation as an immunological abnormality and effectiveness of exercise. Biomolecules. 2019; 9: 223. PubMed: https://pubmed.ncbi.nlm.nih.gov/31181700/

14. Cortesi, Paolo Angelo, Gianluca Trifirò, Simona Serao Creazzola Giovanni Improta, et al. Cost-Effectiveness and Budget Impact of Emicizumab Prophylaxis in Haemophilia A Patients with Inhibitors. Thromb Haemost. 120.02 (2020): 216-228.

PubMed: https://pubmed.ncbi.nlm.nih.gov/31887777/

15. Liesner RJ, Khair K, Hann IM. The impact of prophylactic treatment on children with severe haemophilia. Br J Haematol. 1996; 92: 973-978. PubMed: https://pubmed.ncbi.nlm.nih.gov/8616096/

16. Matsumura Risa. Pharmacokinetics of Extended Half-Life Factor VIII Products By my PKFiTR Is Useful for Personalized Treatment in Children with Severe Hemophilia a. 2019; 1127. 